Supporting Information for

\title{
Interfacial Assembly of Amphiphilic Tiles for Reconfigurable Photonic Surfaces
}

\author{
Jong Bin Kim, Gun Ho Lee, Shin-Hyun Kim* \\ Department of Chemical and Biomolecular Engineering, KAIST, Daejeon, 34141 Republic of \\ Korea \\ *Corresponding author. E-mail: Shin-Hyun Kim (kim.sh@,kaist.ac.kr)
}




\section{SI Contents}

S1. Selective surface modification by directional reactive ion etching

S2. Photonic tiles at air-water interface

S3. Calculation of the pair potential

S4. Influence of RIE treatment on optical property of tiles at the air-water interface

S5. Influence of RIE treatment on the tile alignment

S6. Control of structural color

S7. Evolution of tile assembly during shape transformation

S8. Transfer of tile assembly to soild surface

S9. Description of Movies

S10. Reference

Figure S1. Change of contact angle and surface nanostructure along with RIE treatment. Figure S2. Influence of tile inclination during RIE treatment.

Figure S3. Configuration of tiles at the interface.

Figure S4. Migration of tiles at the convex interface.

Figure S5. Pair interaction for amphiphilic and hydrophilic tiles.

Figure S6. Time-series spectra of the photonic tile at the air-water interface.

Figure S7. Color tuning of photonic tiles.

Figure S8. Behavior of tiles in the shape-transforming air-water interface.

Figure S9. Direct transfer of a tile assembly to a glass substrate.

Figure S10. Inverse transfer of a tile assembly to PDMS.

Movie S1. Iridescence of photonic-tile assembly.

Movie S2. Dynamic undulation of photonic surface.

Movie S3. Shape transformation of photonic surface.

Movie S4. Direct transfer of a photonic-tile assembly to a solid.

Movie S5. Photonic liquid marble. 


\section{S1. Selective surface modification by directional reactive ion etching}

The porous photonic discs and films are treated by reactive ion etching (RIE) with $\mathrm{SF}_{6}$ gas. High directionality of RIE selectively renders the top surface to be hydrophobic through physical etching and fluorination. To make the side walls as well as the top surface of discs hydrophobic, the substrate containing scattered discs in a monolayer on the surface is inclined at $2^{\circ}$ along four different directions during the RIE treatment. The same treatment protocol is used for films to make the same surface property. The duration of RIE treatment is optimized with the films. The duration is varied as $10 \mathrm{~s}, 30 \mathrm{~s}, 60 \mathrm{~s}$, and $120 \mathrm{~s}$ for each inclination and the morphology and waterdrop contact-angle are analyzed (Figure S1). Without RIE treatment, the surface has a hexagonal array of small holes and the contact angle is $57.3^{\circ}$. As the duration increases, so does the contact angle. With the duration of $60 \mathrm{~s} \times 4$, the maximum contact angle of $143.1^{\circ}$ is obtained and no further increase is observed for a longer duration. The contact-angle change is consistent with morphological change. As the duration increases, the uppermost structure gets more open and thinner. With the duration of $60 \mathrm{~s} \times 4$, disconnected nanopillars are formed at the interstitial positions of air cavities, providing a high surface porosity. No significant structural change is made for a duration of $120 \mathrm{~s} \times 4$.

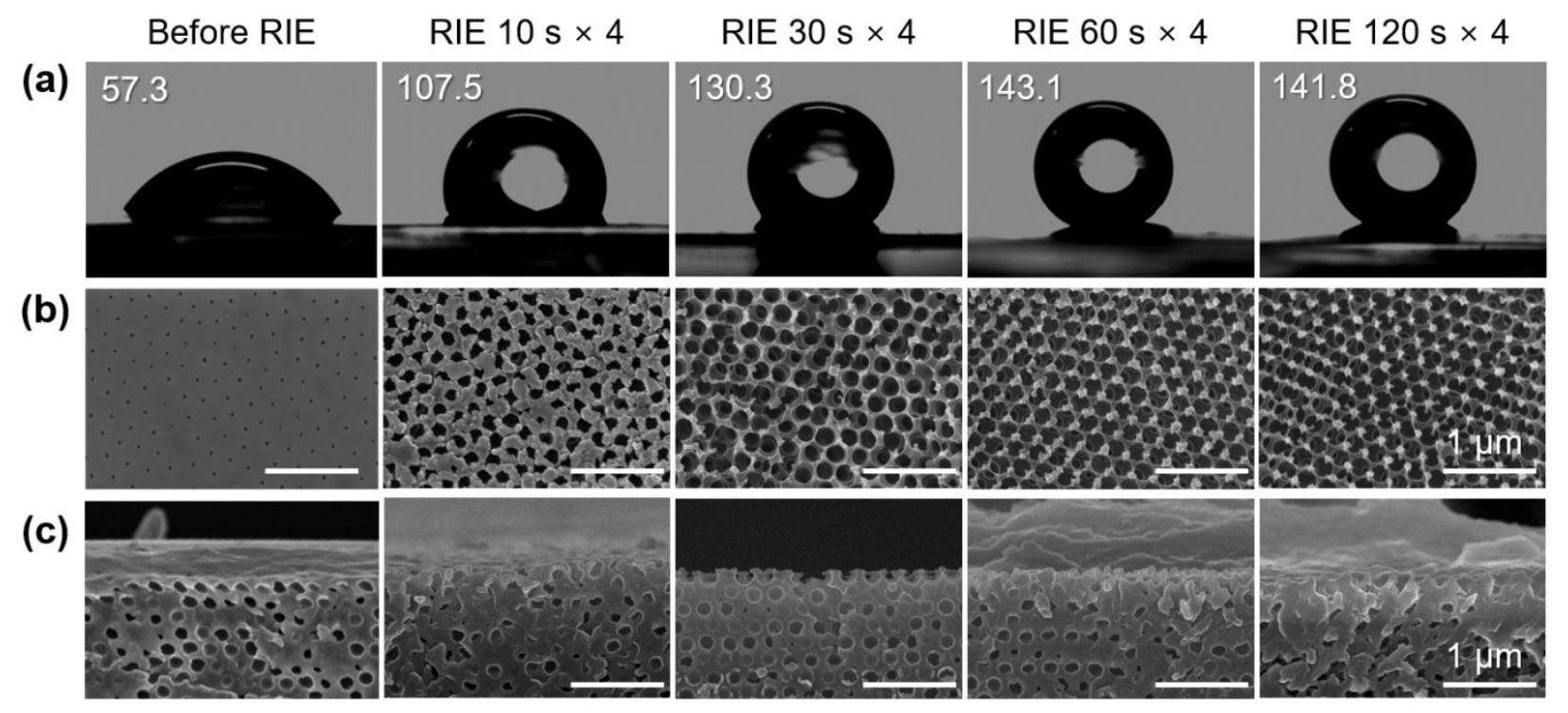

Figure S1. Change of contact angle and surface nanostructure along with RIE treatment. (a) Series of images showing a contact angle of water drop on the RIE-treated of porous films, where the duration of treatment is denoted above each panel. The porous film without the RIE treatment is included in the first column for comparison. (b, c) Series of SEM images showing top surface (b) and cross-section near the top surface (c). 
(a)

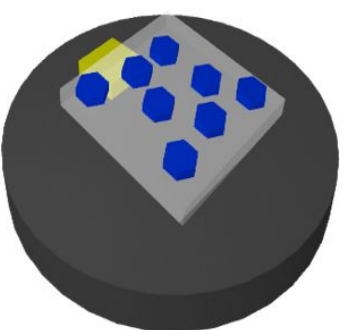

$60 \mathrm{~s}$

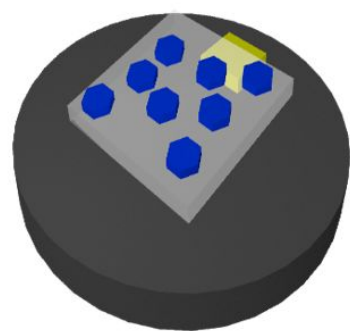

$60 \mathrm{~s}$

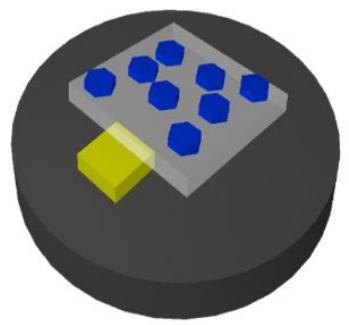

$60 \mathrm{~s}$

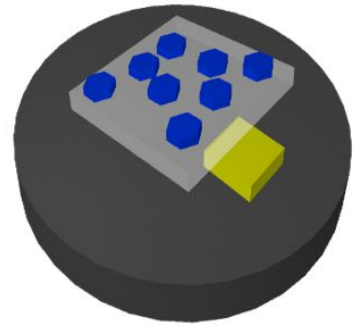

$60 \mathrm{~s}$ (b)

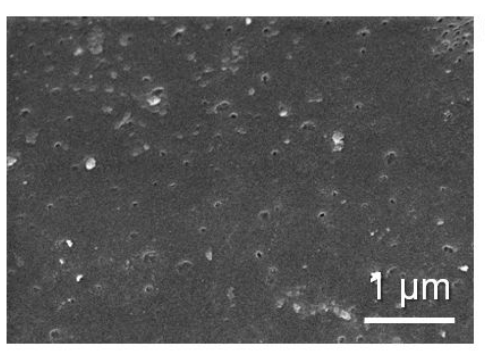

(c)

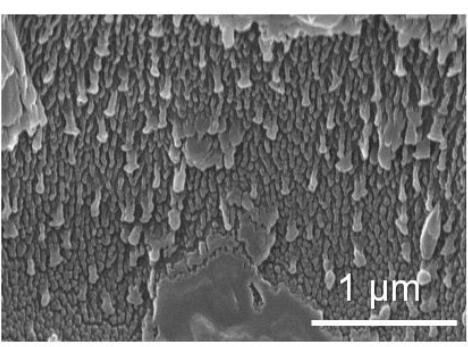

(d)

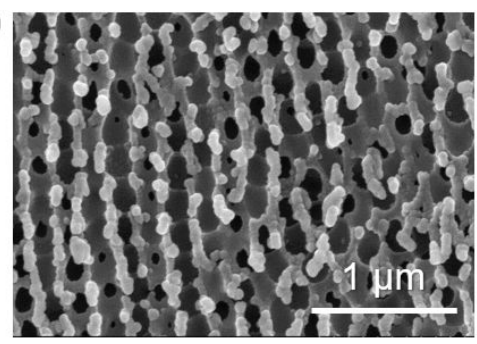

Figure S2. Influence of tile inclination during RIE treatment. (a) Cartoon showing the protocol of RIE treatment with an inclination angle of $2^{\circ}$ along four different directions, where the duration of RIE treatment is set to $60 \mathrm{~s}$ for each direction. (b-d) SEM images of sidewalls of the disc that are untreated (b), RIE-treated without inclination (c), and RIE-treated with the inclination (d). 


\section{S2. Photonic tiles at the air-water interface}

To study the position of the triple line, photonic tiles at air-water interface are transferred into a PDMS block and the surface profiles are analyzed using optical profilometer. To do this, water is replaced with $1 \mathrm{w} / \mathrm{w} \%$ aqueous solution of agarose at $70^{\circ} \mathrm{C}$ by repeating the injection of the solution and suction of the mixture. After gelating the agarose by cooling to room temperature, a PDMS precursor is poured on the surface and cured (Figure S3a and d). After peeling off, the PDMS containing the photonic tiles is subjected to the profile analysis. As the optical profilometer uses the reflection from the surface, it is difficult to directly use water or gel with a small refractive index. The RIE-treated photonic tile shows a weak contrast from the surrounding in the profile (Figure S3b), indicating the body of the tile is exposed to air at the interface. By contrast, the photonic tile without RIE treatment shows a clear profile of the tile after the transfer (Figure S3e), indicating that the body of the tile is fully immersed in the water phase at the air-water interface. That is, the tile with no treatment has the triple line along the top edge, whereas the RIE-treated tile has the triple line along the bottom edge.

(a)

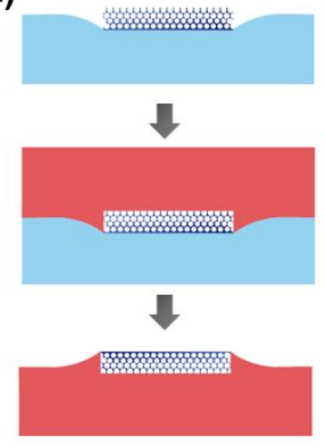

(d)

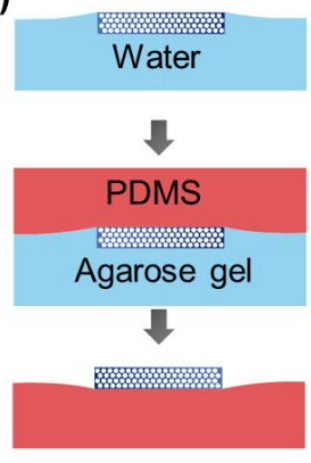

(b)

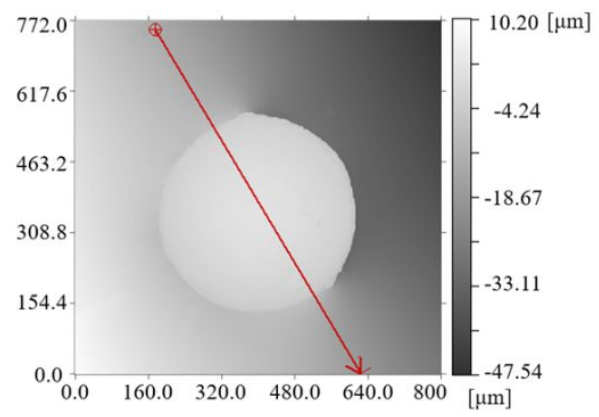

(e)

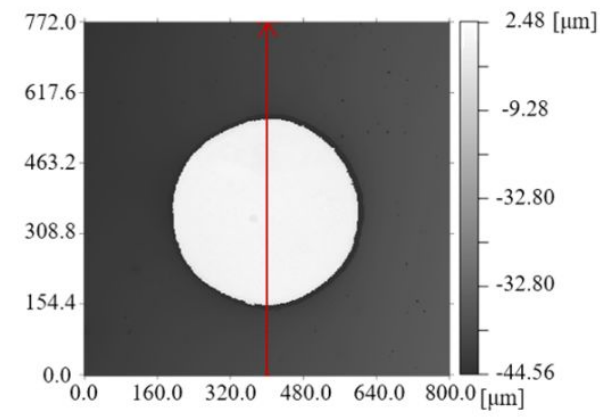

(c)

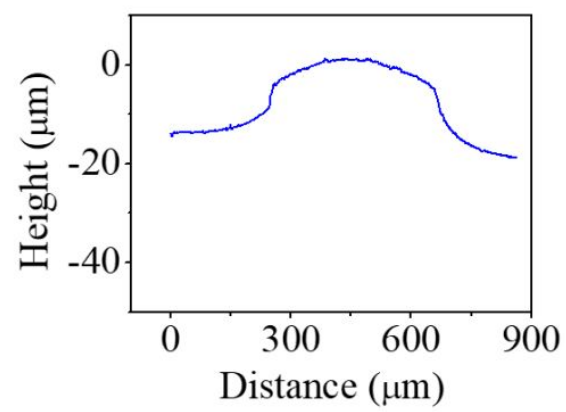

(f)

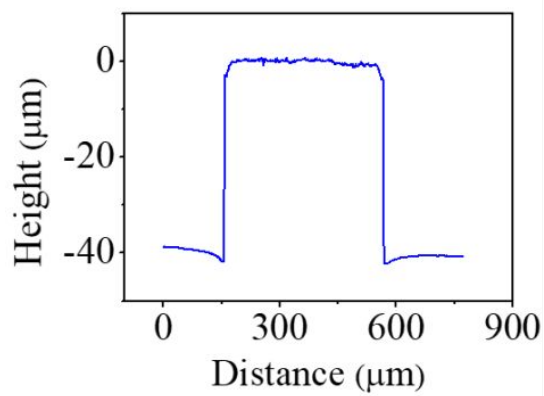

Figure S3. Configuration of tiles at the interface. (a) Cartoons showing the transfer of a RIEtreated photonic tile from the air-water interface to PDMS. (b, c) Gray-scale 2D profile for photonic tile without RIE treatment (b) and profile curve along the straight line denoted in (b) (c). (d-f) The same set for a photonic tile without RIE treatment. 
To further confirm that the position of the triple line, the tiles are anchored at the convex air-water interface (Figure S4a). The RIE-treated tile migrates to the lowest level of the interface (Figure S4b). That is, the tile is surrounded by a medium with a lower density, which is air. By contrast, the tile with no RIE treatment migrates toward the top of the interface (Figure S4c). This indicates that the density of the tile $\left(0.74 \mathrm{~g} / \mathrm{cm}^{3}\right)$ is lower than the surrounding, which is water.

(a)

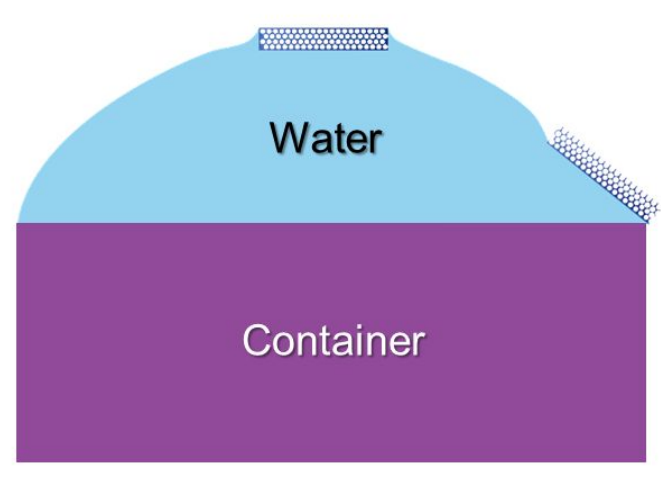

(b)

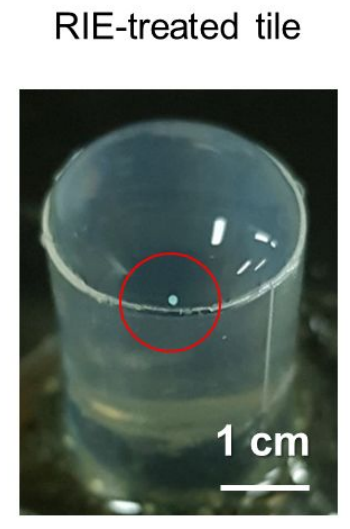

(c) Tile with no RIE treatment

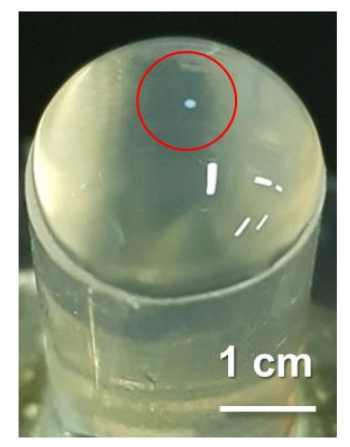

Figure S4. Migration of tiles at the convex interface. (a) Cartoon showing the location of tiles on the convex air-water interface depending on the position of the triple line. (b) Image showing the RIE-treated tile which is located on the bottom. (c) Image showing the tile with no RIE treatment which is located on the top of the interface. 


\section{S3. Calculation of the pair potential}

The motion of photonic tiles is described by Newton's second law:

$m_{d t^{2}}^{d^{2} r}=F_{d r a g}+F_{\text {interaction }}$.

As the inertia and drag forces are estimated from the experimental data for the distance-time relations, the pair-interaction force can be estimated. The center-to-center distance linearly changes with the remaining time until the contact in a log-log plot for RIE-treated amphiphilic tile (Figure S5a). Therefore, $r\left(t_{\max }-t\right)$ fits well to the logarithmic equation:

$$
\log r=\alpha_{1} \log \left(t_{\max }-t\right)+\alpha_{2},
$$

where the fitting yields $\alpha_{1}=0.1613$ and $\alpha_{2}=3.0522$. The hydrophilic photonic tiles with no RIE treatment show nonlinear behavior in the log-log plot (Figure S5a), which fits well to the eighthdegree polynomial:

$$
\mathrm{r}=\mathrm{a}_{8}\left(t_{\max }-t\right)^{8}+\mathrm{a}_{7}\left(t_{\max }-t\right)^{7}+\ldots+a_{0},
$$

where the fitting yields $a_{8}=7.7153 \times 10^{-6}, a_{7}=-2.8540 \times 10^{-4}, a_{6}=0.00187, a_{5}$ $=0.06087, \mathrm{a}_{4}=-1.335, \mathrm{a}_{3}=11.61, \mathrm{a}_{2}=-53.56, \mathrm{a}_{1}=161.95$, and $\mathrm{a}_{0}=424.8$.

The drag force is proportional to relative velocity, medium viscosity, and the dimension of the tile, according to the Stokes' law. The drag coefficient is separately estimated for the RIEtreated amphiphilic tile and hydrophilic tiles with no treatment due to the different position of the triple line. As the RIE-treated tile has a triple line at the bottom edge, the drag coefficient is estimated by halving the coefficient for a disc with zero thickness fully immersed in a medium: ${ }^{1}$

$$
\mathrm{F}_{\text {drag, amphiphilic tile }}=\frac{1}{2} \mathrm{~F}_{\text {drag, zero }- \text { thickness }}=\frac{8}{3} \mu V d,
$$

where $\mu$ is the viscosity, $V$ is velocity $(=\mathrm{d} r / \mathrm{d} t)$, and $d$ is a diameter of the tile. By contrast, the hydrophilic tile with no RIE treatment has a triple line at the top edge. The drag coefficient for the hydrophilic tile is estimated by halving the coefficient for a disc with double thickness fully immersed in a medium:

$$
\mathrm{F}_{\text {drag, hydrophilic tile }}=\frac{1}{2} \mathrm{~F}_{\text {drag, double thickness }}=\frac{8}{3} K \mu V d .
$$

The value of $K$ is determined by the following equations:

$$
K=A+B\left(\frac{2 l}{d}\right)^{C}, A=1+a\left(\frac{d}{l_{b}}\right)^{b}, B=\left(f+g \frac{d}{l_{b}}\right)^{2}, \text { and } C=1-k e^{-p\left(d / l_{b}\right)},
$$

where $a=0.8160, b=1.0754, f=1.0418, g=1.3312, k=0.2269$, and $p=1.51$. The thickness of the tile, $l$, is set to $50 \mu \mathrm{m}$. The distance of tile to the immobile parallel boundary, $l_{\mathrm{b}}$, is set to $2 \mathrm{~mm}$ by considering the depth of water.

Pair potential, $U(r)$, can be calculated from the equation (S1) using the relation: 


$$
\mathrm{F}_{\text {interaction }}=-\frac{\partial U}{\partial r}
$$

The equation (S1) is modified as follows:

$$
\frac{\partial U}{\partial r}=\frac{8}{3} K \mu d \frac{d r}{d t}-m \frac{d^{2} r}{d t^{2}}
$$

where $K=1$ for the RIE-treated amphiphilic tile. The mass of tile, $m$, is set to $6.35 \times 10^{-9} \mathrm{~kg}$. Pair potential, $U(r)$, is obtained by integrating equation (S8):

$$
U(r)=U\left(r_{\text {contact }}\right)+\int \frac{8}{3} \mu d\left(\frac{d r}{d t}\right)^{2} d t-\int m \frac{d^{2} r}{d t^{2}}\left(\frac{d r}{d t}\right) d t
$$

The magnitude of the potential energy for the amphiphilic tile is much larger than that for the hydrophilic tile (Figure S5b). The pair potential rapidly drops as the distance decreases for both RIE-treated amphiphilic tile and hydrophilic tile with no treatment. However, the distance dependence is different. To roughly quantify the difference, we assume that $U(r)$ follows the relation:

$$
U(r) \sim r^{\beta},
$$

where the value of $\beta$ is obtained from the slope of $U(r)$ in a log-log plot (Figure $2 \mathrm{~h}$ ). Although the curve is not perfectly linear especially for the hydrophilic tile, we extract an average slope for a simple comparison of the distance dependence of potential energy. The value of $\beta$ is -5.5 for the amphiphilic tiles and -4.0 for hydrophilic tiles, indicating the amphiphilic tiles have a stronger dependence on the distance.

(a)

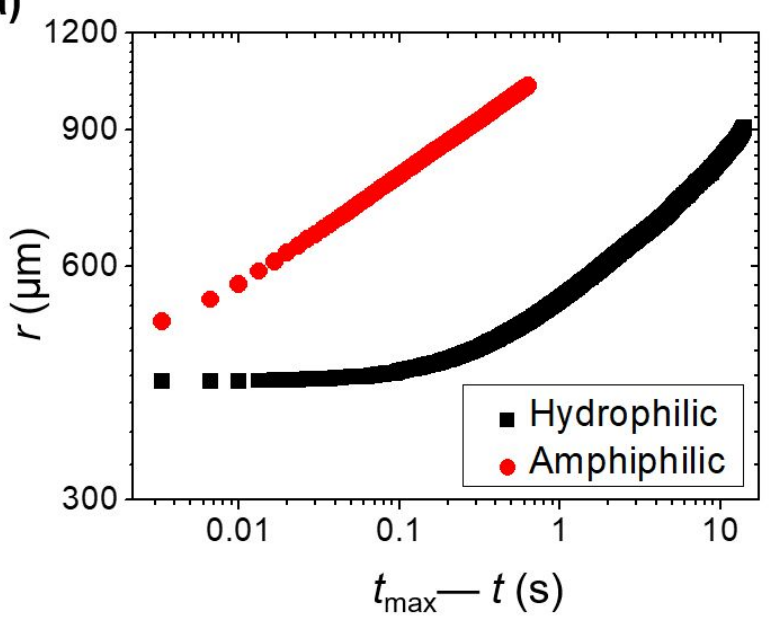

(b)

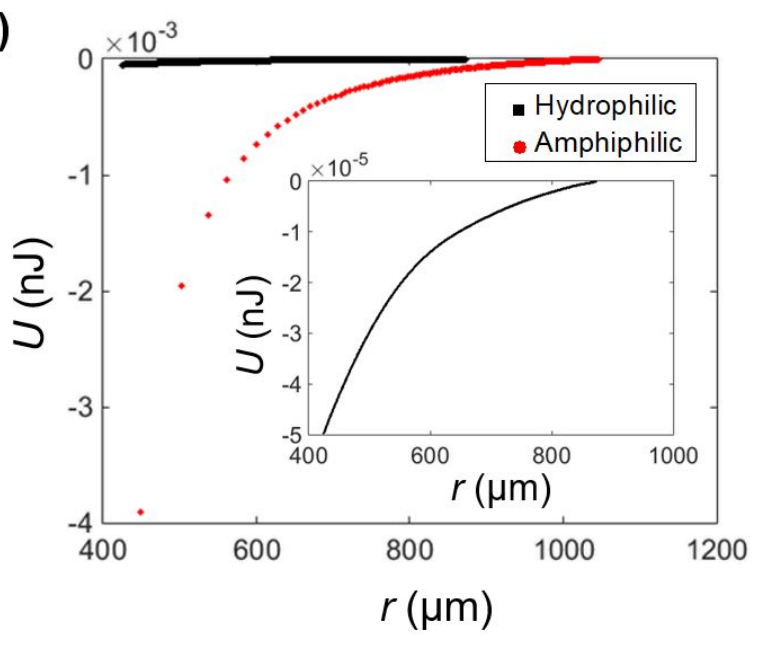

Figure S5. Pair interaction for amphiphilic and hydrophilic tiles. (a) The center-to-center distance as a function of the remaining time until the contact, $r\left(t_{\max }-t\right)$, in a log-log plot for amphiphilic tile (red circles) and hydrophilic tile (black squares). (b) Pair potentials for two different tiles. The inset is the pair potential for hydrophilic tiles. 


\section{S4. Influence of RIE treatment on optical property of tiles at the air-water interface}

The RIE treatment makes a stark difference in the position of the triple line, as confirmed in Figure S3 and S4. In addition, it influences a long-term optical property of the tiles anchored at the airwater interface. The RIE-treated photonic tiles show consistent reflectance spectra and color at least for 15 days when they are anchored at the air-water interface (Figure S6a). By contrast, the tiles without RIE treatment show a slight change in the shape of the reflectance spectrum over time although the change is small and no color change is observed. This change is attributed to the partial invasion of water into the pores of photonic tiles.

(a)

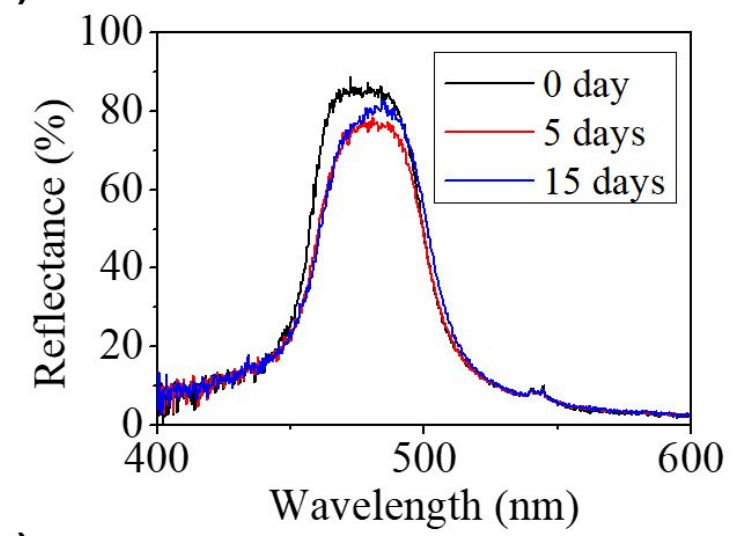

(b)

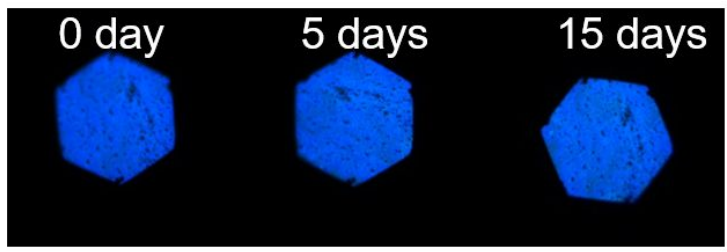

(c)

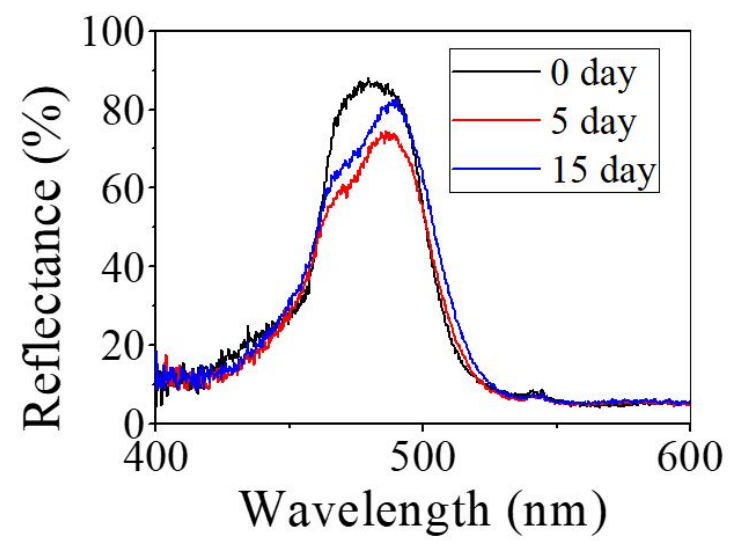

(d)

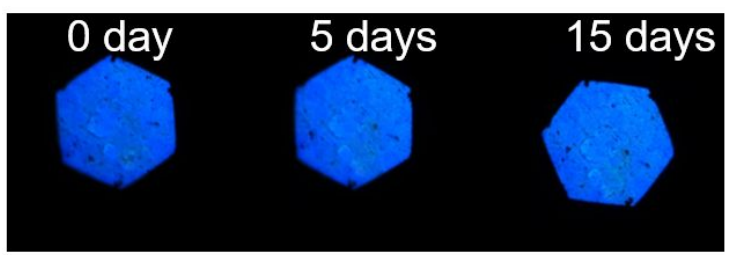

Figure S6. Time-series spectra of the photonic tile at the air-water interface. (a, b) Reflectance spectra and OM images of the RIE-treated photonic tile anchored at the air-water interface for the denoted times. The images are taken with the field stop. (c, d) The same set for the tile with no RIE treatment. 


\section{S5. Influence of RIE treatment on the tile alignment}

The RIE treatment makes almost all the amphiphilic photonic tiles sit on the air-water interface with a planar alignment. When the tiles without the RIE treatment are applied on the interface, approximately $10 \%$ of the tiles stand upright, which perturb the ordering.

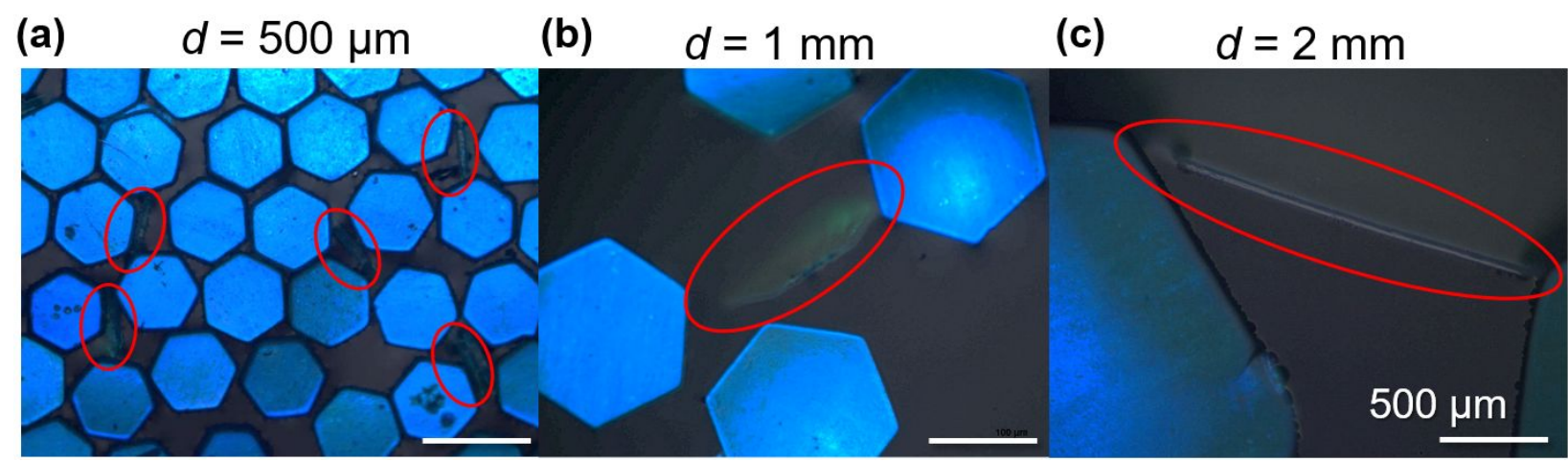

Figure S7. OM images showing the assembly of photonic tiles with no RIE treatment at the airwater interface, where the dimensions of the tiles are $500 \mu \mathrm{m}(\mathrm{a}), 1 \mathrm{~mm}$ (b), and $2 \mathrm{~mm}$ (c), respectively. The red ellipses denote the tiles inclined. 


\section{S6. Control of structural color}

The structural color or stopband position can be easily tuned by varying the diameter of silica particles according to the equation (1). For example, the photonic tiles made from silica particles with a diameter of $D=178 \mathrm{~nm}$ show a blue color and reflectance peak at $485 \mathrm{~nm}$ (Figure 1), whereas those from silica particles with $D=215 \mathrm{~nm}$ show an orange color and reflectance peak at $592 \mathrm{~nm}$ (Figure S8). The reflectance peak positions are in good agreement with equation (1).

(a)

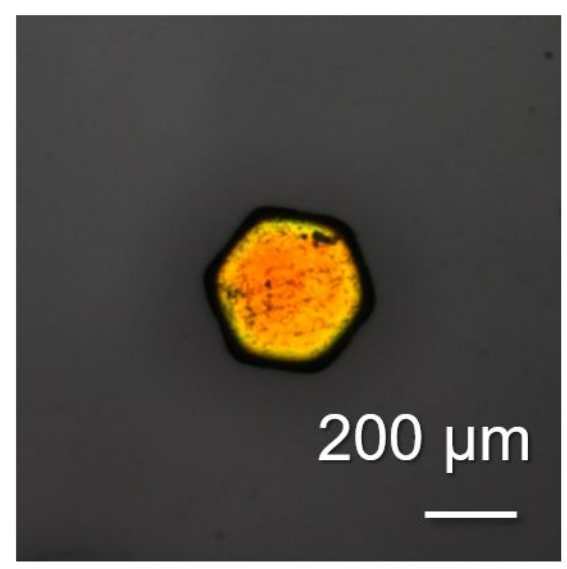

(b)

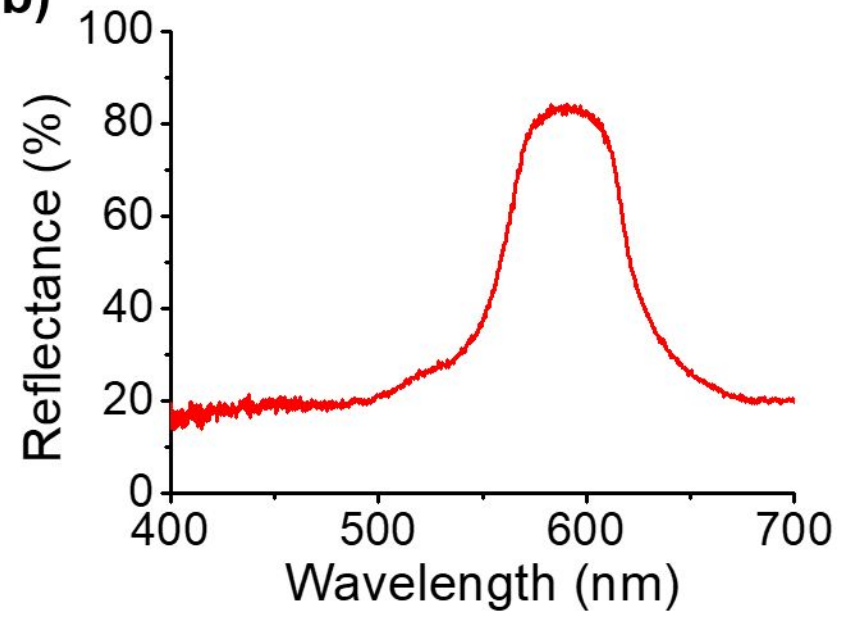

Figure S8. Color tuning of photonic tile. (a, b) OM image (a) and reflectance spectrum (b) of orange-colored photonic tiles made from silica particles with a diameter of $215 \mathrm{~nm}$. 


\section{S7. Evolution of tile assembly during shape transformation}

The shape of a flexible container is transformed to make shape-reconfigurable photonic surfaces. The container has rounded corners when it is transformed to a square and triangle (Figure $3 \mathrm{f}$ ). While maintaining the length of the container perimeter, the relative areas of the square and triangle to the original circle are calculated as 0.93 and 0.91 , where the radii of rounded corners are used from photographs of the containers (Figure S9a). Because the tiles are strongly adsorbed, the interface is undulated during the shape transformation. At the early stage, the interface is locally undulated; the undulated regions appear dark due to the violation of reflection condition. As the container is further transformed, the interface rises due to the shrinkage of the cross-section, which causes the interface to be a mesa-shaped. The middle of the mesa is composed of a wellordered array of tiles, whereas the edge is curved downward. This mesa-shaped interface results in a bright reflection on the most area of the interface except the vicinity of the wall. This process is shown in the series of images and cartoons (Figure S9b, c). When the shape is changed from a square or triangle to a circle, the original array of tiles is recovered (Figure S9d).

(a)
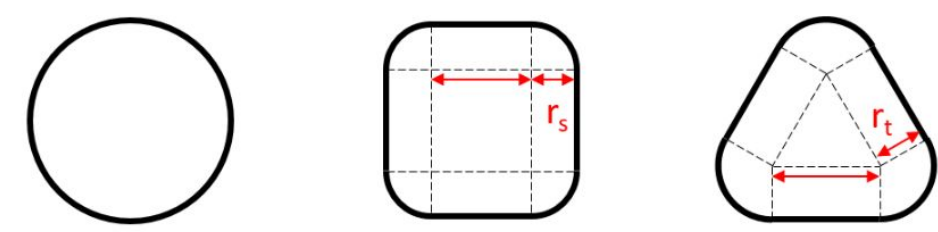

(b)
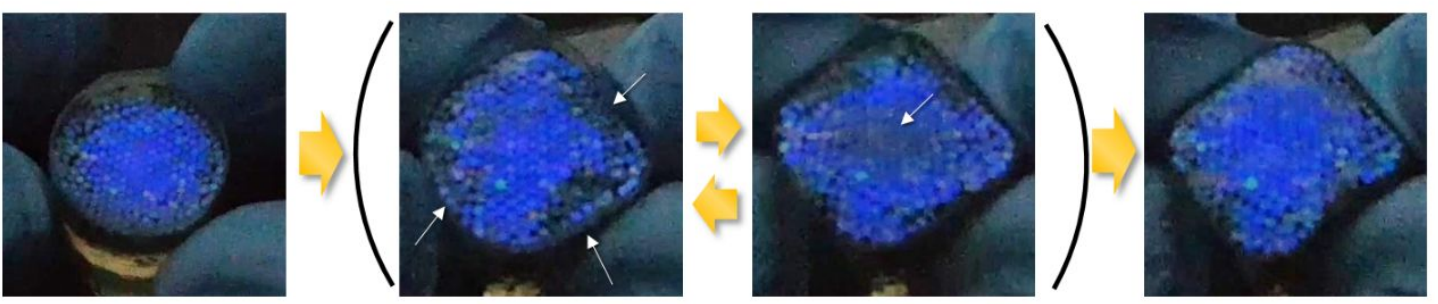

(c)
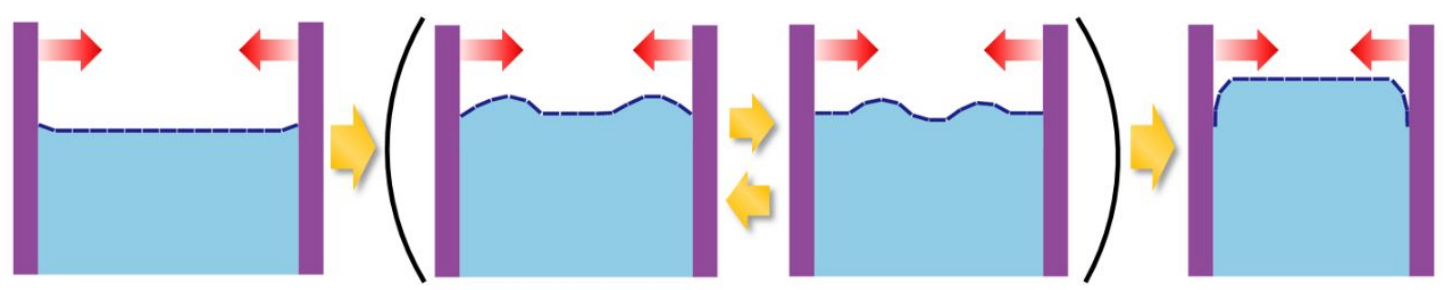

(d)
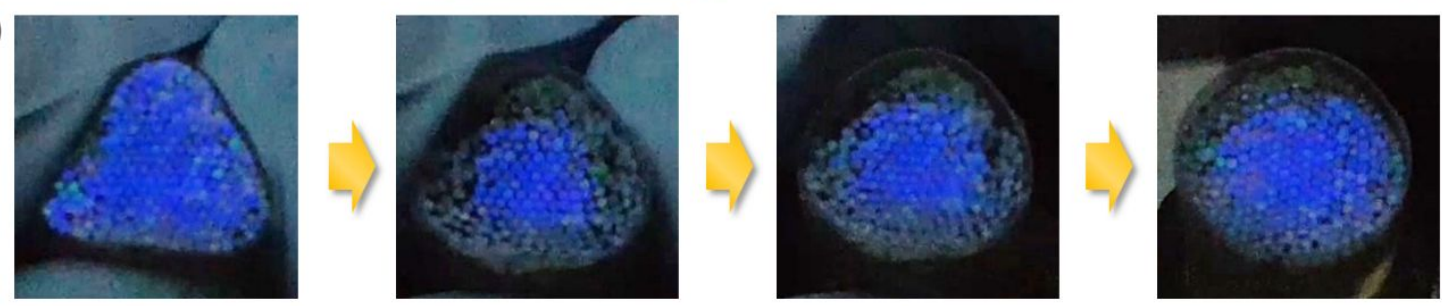

Figure S9. Reconfigurable shape of interface. (a) Model shapes of containers constructed from experimental observation, where radii of rounded corners are denoted with $r_{\mathrm{s}}$ and $r_{\mathrm{t}}$ for square and triangle. (b) Series of images showing the evolution of tile-anchored interface during the transformation from a circle to square. (c) Series of cartoons showing the evolution of interface at the side view. (d) Series of images showing the recovery of tile array for transformation from a triangle to circle. 


\section{S8. Transfer of tile assembly to solid surface}

The photonic tiles with orange reflection colors are assembled at the air-water interface, which is also directly transferred onto a glass substrate by the Langmuir-Blodgett method (Figure S10). The resulting photonic coating shows the angle-dependent color. For example, the coatings are green when observed with an angle (Figure S10b).

(a)

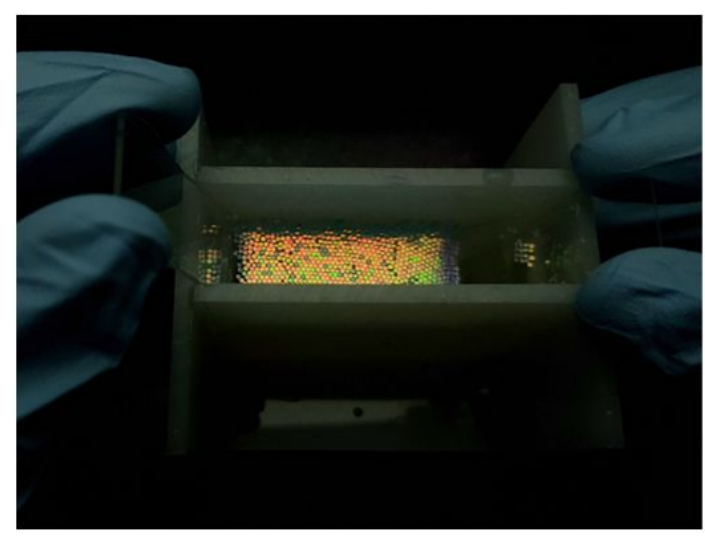

(b)

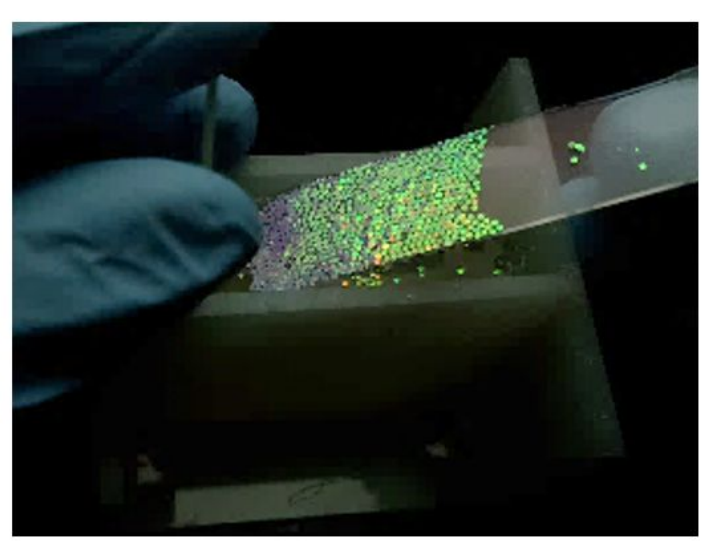

Figure S10. Direct transfer of a tile assembly to a glass substrate. (a) Interfacial assembly of photonic tiles with orange colors made from silica particles with $D=215 \mathrm{~nm}$. (b) Photonic coating transferred from the interface by Langmuir-Blodgett method, where the color appears green due to the angle of observation.

The photonic coating can be inversely transferred on a PDMS elastomer. Amphiphilic photonic tiles are first assembled at the air-water interface and then the water phase is replaced to $0.6 \mathrm{w} / \mathrm{w} \%$ agarose solution at $70^{\circ} \mathrm{C}$. The agarose is gelated at the room temperature, on which PDMS is poured and then thermally cured at the room temperature for 3 days. During the curing, water in the gel is partially evaporated, which causes small undulation of the interface. Afterward, the PDMS is peeled off from the gel, forming a flexible photonic surface. 


\section{(a)}

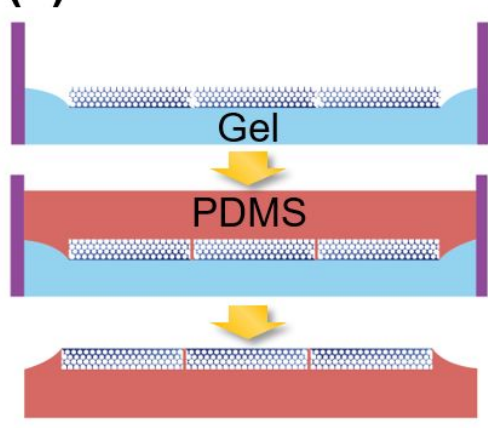

(b)

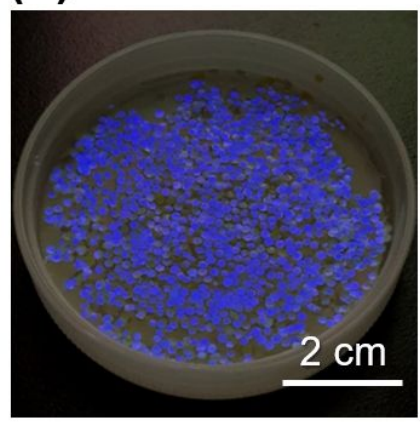

(c)

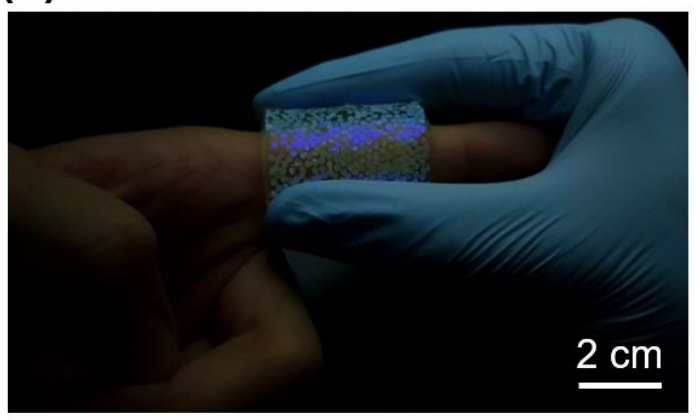

(d)

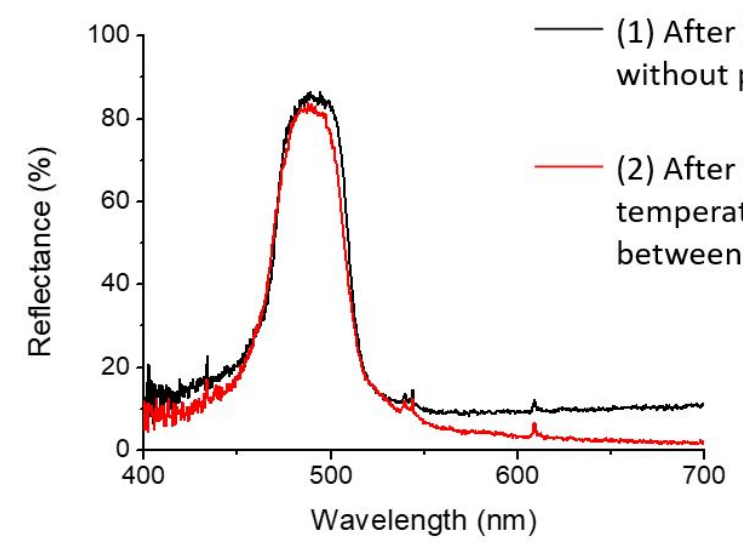

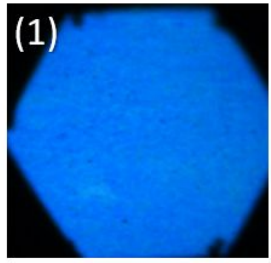

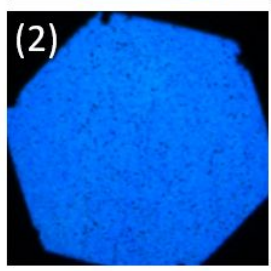

Figure S11. (a-c) Cartoons and images showing the transfer onto PDMS film. The water is first gelated, over which a PDMS precursor is poured and cured (b). The PDMS film is peeled off, which can be rolled around a finger (c). (d) Reflectance spectra and OM images of photonic tile at the air-gel interface and that transferred on PDMS elastomer. There is no shift of reflectance peak or reflection color, indicating no infiltration of PDMS into the pores of tiles. 


\section{S9. Description of Movies}

Movie S1. Angle-dependent color change of a photonic-tile assembly for specular reflection.

Movie S2. Dynamic reconfiguration of a photonic-tile assembly for interface undulation (32x slower than real-time).

Movie S3. Dynamic rearrangement of photonic tiles during the shape transformation of the container $(2 \times$ faster than real-time).

Movie S4. Transfer of a tile assembly from an air-water interface to a glass substrate using Langmuir-Blodgett method.

Movie S5. Formation of liquid marble using amphiphilic photonic tiles.

\section{S10. Reference}

1. Trahan, J. F. Stokes drag on a thin circular disk moving edgewise midway between parallel plane boundaries. J. Fluids Eng. 2009, 128, 887-889. 